\title{
Vapor-Based Polymer Gradients
}

\author{
Yaseen Elkasabi, Joerg Lahann*
}

Chemical vapor deposition (CVD) co-polymerization was used to fabricate polymer coatings, which comprise of reactive surface composition gradients. Two functionalized derivatives of [2.2]paracyclophane were fed into a two-source CVD system at a $180^{\circ}$ angle, then copolymerized and deposited as a polymer gradient. Infrared and X-ray photoelectron spectroscopy (XPS) confirmed the compositional changes within the bulk polymer and at the surface. By manipulating process parameters, gradients of tailored compositional slope can be deposited on a wide range of substrates. We also were able to selectively immobilize fluorescence-labeled ligands onto the reactive polymer gradients, making CVD-based gradient surfaces a flexible platform for fabricating biomolecular substrates.

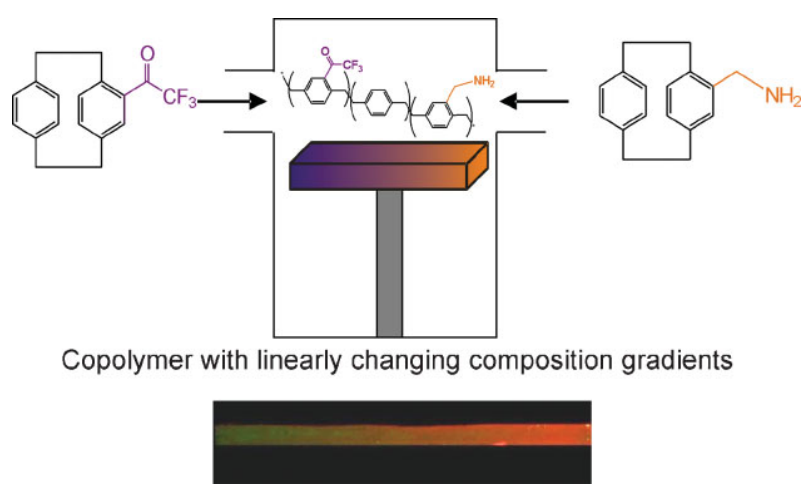

\section{Introduction}

In nature, biological information is typically encoded and displayed in the form of continuous gradients mitigating important cellular events, such as cell signaling, cell development, or chemotaxis. Mimicking surfaces with natural, spatially continuous gradients is therefore of decisive importance to a range of biological applications including studies of neuronal growth and differentiation, ${ }^{[1-3]}$ the design of cell migration ${ }^{[4-6]}$, and inflammation $^{[7]}$ assays, microfluidic cell culture, ${ }^{[8]}$ or discoverydriven biomaterials research. ${ }^{[9,10]}$ In spite of the importance of surface gradients for biological applications, their realization, especially with biomedically relevant polymers, has been challenging. Methods of making gradients are often relatively undifferentiated and are potentially associated with a number of limitations: (i) Translation of

J. Lahann, Y. Elkasabi

Departments of Chemical Engineering, Material Science and Engineering, and Macromolecular Science and Engineering, University of Michigan, 2300 Hayward Street, Ann Arbor, Michigan 48109, USA

Fax: +(734) 764 7453; E-mail: lahann@umich.edu chemical gradients into biological gradients requires immobilization of biomolecules, which is often not possible with existing gradient materials. ${ }^{[11,12]}$ (ii) Due to technical constraints, such as chemical resolution, resulting gradients are often intrinsically discontinuous with potentially steep transitional steps. ${ }^{[13-15]}$ (iii) Methods for making gradients are in many cases restricted to a particular surface chemistry, such as thiols to gold or siloxanes to glass or silicon. (iv) As a consequence, the range of materials, to which a given protocol can be applied, may become rather limited. ${ }^{[16,17]}$ Although a range of methods have been developed that have the potential to address some or all of these challenges, experimental deficiencies are still in gross discrepancy to the rather elaborated approaches that have been developed over the last two decades for inorganic surface gradients. Therein, the use of vapor-based fabrication methods for inorganic materials resulted in efficient, high-throughput screening for catalytic activity, conductivity, or luminescence. ${ }^{[18]}$ Where fabrication of gradients in inorganic materials has greatly benefited from anisotropic chemical vapor deposition (CVD), ${ }^{[18]}$ polymer gradients have been so far prepared by solutionbased processes, such as bulk diffusion, ${ }^{[11,19]}$ microfluidic 
pathways, ${ }^{[13,14]}$ lithography, ${ }^{[15,16,20]}$ or combinations thereof. ${ }^{[12,17]}$

By taking advantage of vapor-based polymers, we have now established a simple method for polymer gradients with continuously changing surface compositions. Given the wide-range applicability of CVD polymerization to a diverse set of different substrates, ${ }^{[21]}$ polymer gradients based on CVD polymer technology have the potential to expedite biomaterials discovery, much like it has been witnessed for inorganic materials over the past decades. ${ }^{[22]}$

Our approach toward polymer gradients takes advantage of CVD polymerization of substituted [2.2]paracyclophanes to prepare polymer coatings with identical backbone chemistries, but different functional side groups. ${ }^{[21]}$ Compared to plasma polymerization, which has been previously used to prepare polymer gradients, ${ }^{[23]}$ CVD polymerization of functionalized poly ( $p$-xylylene) (PPX) has the potential to create robust, chemically well-defined polymers with a diverse range of reactive side groups typically without appreciable side reactions. Recently, copolymerization of two different [2.2]paracyclophanes resulted in surfaces with fine-tunable composition ratios. ${ }^{[24]}$ With the concept of CVD co-polymerization demonstrated, the fundamental feasibility of a vaporbased gradient fabrication tool that mimics the processes developed for inorganic materials discovery is now within the reach of soft materials.

\section{Experimental Part}

\section{CVD Co-Polymerization}

CVD depositions were carried out in a custom-built CVD system, equipped with two inlet sources. Each source consisted of a quartz tube encased in a three-zone tube furnace, and both tubes entered the deposition chamber directly opposite to each other. 4trifluoroacetyl[2,2]paracyclophane (1) was synthesized using an established synthesis route. ${ }^{[25]}$ 4-Aminomethyl[2,2]paracyclophane (2) was purchased from Uniglobe Kisco, (White Plains, NY) and used as received. Starting materials $\mathbf{1}$ and $\mathbf{2}$ were loaded separately into two feed dishes, with each dish loaded into its own source. A system pressure of 0.165 Torr and sublimation temperatures between 80 and $110^{\circ} \mathrm{C}$ were used to ensure sublimation. Argon carrier gas was used to independently control the flow velocities of sublimated dimers (1) and (2). Three different process conditions produced gradients with differing compositional rates of change. Condition $1: M_{1}=160 \mathrm{mg}, M_{2}=205 \mathrm{mg}$, $\mathrm{Ar}_{1}=6.5 \mathrm{sccm}, \mathrm{Ar}_{2}=32 \mathrm{sccm}$. Condition $2: \mathrm{M}_{1}=200 \mathrm{mg}, \mathrm{M}_{2}=205$ $\mathrm{mg}, \mathrm{Ar}_{1}=7.2 \mathrm{sccm}, \mathrm{Ar}_{2}=29.7 \mathrm{sccm}$. Condition 3: $\mathrm{M}_{1}=120 \mathrm{mg}$, $\mathrm{M}_{2}=205 \mathrm{mg}, \mathrm{Ar}_{1}=5.7 \mathrm{sccm}, \mathrm{Ar}_{2}=35 \mathrm{sccm}$. The sample holder was set four inches below the position of both sources. To ensure gradient formation, the sample holder was not rotated. Although the sample holder remained at $15^{\circ} \mathrm{C}$, a copper plate was set on top of the sample holder, in order to control the sample's angle of inclination. Deposition occurred on silicon, gold, and glass substrates. To minimize wall deposition, the chamber wall was heated to $120^{\circ} \mathrm{C}$.

\section{Surface Characterization}

X-ray photoelectron spectroscopy (XPS) was performed on CVDcoated silicon substrates $(1 \mathrm{~cm} \times 1 \mathrm{~cm})$. XPS data were recorded on an Axis Ultra XPS (Kratos Analyticals, UK) equipped with a monochromatized AlK $\alpha \mathrm{X}$-ray source. All spectra were calibrated with respect to the nonfunctionalized aliphatic carbon with a binding energy of $285.0 \mathrm{eV}$ and were corrected for atomic sensitivity factors. Thicknesses were recorded at a wavelength of $532 \mathrm{~nm}$ using an $\mathrm{EP}^{3}-\mathrm{SW}$ imaging ellipsometer (Nanofilm Technologie GmbH, Germany). Four-zone nulling experiments were performed at an angle of incidence of $70^{\circ}$, and an anisotropic Cauchy model was used to model the ellipsometric parameters $\psi$ and $\delta$. Infrared spectroscopy was performed on a Nicolet 6700 spectrometer utilizing the grazing angle accessory (Smart SAGA) at a grazing angle of $85^{\circ}$. FTIR spectra were corrected for any residual baseline drift. Au-coated Si substrates were used for FTIR measurements, using blank $\mathrm{Au}$-coated $\mathrm{Si}$ as a reference.

\section{Surface Modification}

Surface reactions were performed on CVD-coated glass microscope slides ( $3^{\prime \prime} \times 1^{\prime \prime}$, Fisher Inc.). Biotin hydrazide and rhodamine-linked streptavidin were purchased from Pierce Inc., and Atto 655 NHS ester was purchased from Sigma-Aldrich. All surface reaction experiments were performed on glass substrates. A PDMS membrane was molded and cut in the shape of a long rectangle made for holding fluid within its boundaries. The membrane was placed on the film and allowed to seal, and the reaction buffer filled the membrane. Then the entire slide was immersed in a particular washing buffer, with the PDMS still remaining on the surface. Reactions were carried out in a closed Petri dish, to prevent the reaction mixtures from drying out. All surfaces were rinsed with DI water after applying the ligands and using the washing buffers. Consecutive surface reactions were conducted at each location. Fluorescence images and intensities were acquired using a GenePix 4000B scanner with $532 \mathrm{~nm}(17 \mathrm{~mW})$ and $635 \mathrm{~nm}$ (10 $\mathrm{mW}$ ) lasers. Both excitation wavelengths were scanned simultaneously at $100 \mu \mathrm{m}$ spatial resolution. Colors shown in the scanning images were not true colors, but were set for best visualization.

\section{Ligand 1}

$0.5 \mathrm{ml}$ of anhydrous dimethylformamide was added to $1 \mathrm{mg}$ Atto $655 \mathrm{NHS}$ ester. From this, $5.0 \mu \mathrm{l}$ were diluted with $7 \mathrm{ml} 0.1 \mathrm{M}$ sodium bicarbonate buffer ( $\mathrm{pH} 8.3$ ), containing $0.02 \% \mathrm{v} / \mathrm{v}$ Tween 20. NHS esters are time-sensitive in aqueous solution, so the buffer was applied to the film immediately upon dilution. The solution was incubated for $1 \mathrm{~h}$ at room temperature, after which the surface was rinsed with ethanol for $10 \mathrm{~min}$ and with PBS/Tween solution for $30 \mathrm{~min}$.

\section{Ligand 2}

Biotin hydrazide was diluted to $10 \times 10^{-3} \mathrm{M}$ in phosphate buffered saline (PBS pH 7.4). The solution was acid-catalyzed (HAc) prior to 
surface application. Incubation time lasted 7-10 min, which was followed by a wash of PBS containing $0.02 \% \mathrm{v} / \mathrm{v}$ Tween 20 . The area was then applied with rhodamine-linked streptavidin solution $(0.075 \mathrm{mg} \cdot \mathrm{ml}$ in PBS; $0.02 \% \mathrm{v} / \mathrm{v}$ Tween $20 ; 0.1 \% \mathrm{w} / \mathrm{v}$ bovine serum albumin) for $15 \mathrm{~min}$. Finally, the entire glass slide was immersed for $1 \mathrm{~h}$ in a PBS/Tween/BSA solution.

\section{Results and Discussion}

The experimental setup for gradient co-polymerization of functionalized [2,2]paracyclophanes is illustrated in Figure 1. Instead of the traditional single-source CVD system, ${ }^{[26]}$ a polymerization chamber with two sources at a $180^{\circ}$ angle has been designed. Each source can independently provide different types of starting materials. During CVD co-polymerization using dual sources, each ring-constrained [2.2]paracyclophane is thermally converted into corresponding quinodimethanes and transferred into the reaction chamber. Next, the quinodimethanes spontaneously co-polymerize below a certain threshold temperature onto a temperature-controlled substrate (typically $40-60^{\circ} \mathrm{C}$ depending on the chemical structure of the [2.2]paracyclophane). While the monomer flows over a substrate, gradual depletion of the monomer from the gas phase occurs, resulting in a gradual decrease in polymer composition of the vapor phase. If two monomers are deposited countercurrently to each other, each monomer's concentration should decrease with increase in distance from the respective source. As a consequence, the relative ratio of the components, which make up the polymer film, will form a continuous gradient. In order for gradient deposition to occur in this manner, however, the mass transport properties of both monomers must allow for sufficient manipulation. Some process variables that can affect the gradient formation include system pressure, argon flow rates, substrate temperature, sample height, monomer loading, and pyrolysis temperatures. The CVD-based gradient experiments were conducted in a vacuum chamber, which accommodates a $30.5 \mathrm{~cm}$ diameter sample holder placed between the two source inlets. Starting material 4trifluoroacetyl-[2.2]paracyclophane (1) was loaded into source 1, while 4-aminomethyl-[2.2]paracyclophane (2) was loaded into source 2. Pyrolysis temperatures of $670^{\circ} \mathrm{C}$ were used in both source systems. Under these conditions, paracyclophanes $\mathbf{1}$ and $\mathbf{2}$ were converted into the corresponding quinodimethanes $\mathbf{3}$ and $\mathbf{4}$. Deposition rates were adjusted by controlling the sublimation rates of starting material $\mathbf{1}$ and $\mathbf{2}$, so that sublimation of both dimers started and finished simultaneously after a $10 \mathrm{~min}$ period. Dimer sublimation rates were controlled by changing the amount of loaded starting material, while keeping sublimation temperatures and pressure constant. In addition, rapid venting of the deposition chamber with argon after CVD deposition improved gradient quality.

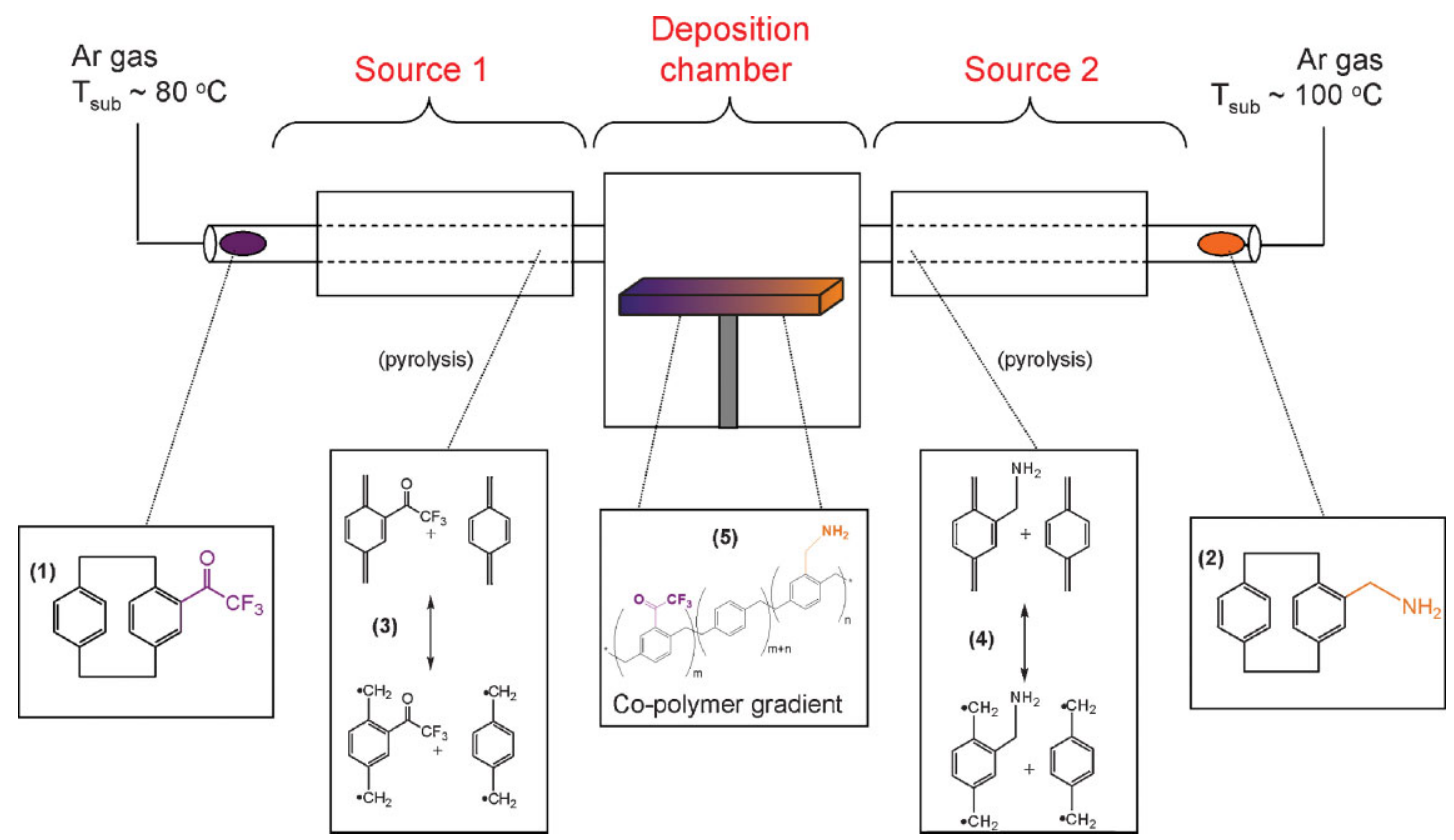

Figure 1. A side-view schematic of the custom-built two-source CVD system. Each source consists of a quartz tube that passes through a three-zone furnace and then connects into the deposition chamber. Both the tubes and the chamber together are held at 0.16 Torr. (1) and (2) sublimate, undergo pyrolysis, and then copolymerize to deposit on the sample holder. The specified process conditions create a PPX film possessing a functional composition gradient. 
Gradient co-polymerization yielded continuous polymer films with thicknesses varying between 120-190 nm. Thickness differences were occasionally observed along the polymer gradient, but did not compromise the chemical composition gradients or the gradient reactivity.

Steady gradient formation with respect to bulk composition across the polymer films was evaluated using a combination of surface analytical methods. Fourier transform infrared (FTIR) spectroscopy was chosen because of its ability to detect relative changes in characteristic bond vibrations. The trifluoroacetyl functional group of poly(4trifluoroacetyl-p-xylylene-co-p-xylylene) resulted in characteristic vibrational modes at $1716 \mathrm{~cm}^{-1}$ associated with the carbonyl group and at 1 200, 1 152, and $973 \mathrm{~cm}^{-1}$ (C-F stretches). Aminomethyl groups have characteristic $\mathrm{N}-\mathrm{H}$ and C-N signals at $3368,3302,1640$, and $829 \mathrm{~cm}^{-1}$, respectively. During CVD co-polymerization, gold substrates were placed at specific positions along the length of the $12^{\prime \prime}$ diameter sample holder, and the polymer coating was deposited using the two-source system (Figure 1). Relative ratios of vibrational signal intensities associated with trifluoroacetyl and aminomethyl groups were determined for each substrate. Figure 2 shows IR spectra at various locations along the sample holder. A detailed FTIR analysis of these samples revealed a transition from strong trifluoroacetyl signals to strong aminomethyl signals, most evidently from the band vibrations at 973 and $829 \mathrm{~cm}^{-1}$. Correlation between FTIR and XPS data provides access to polymer compositions at the surface. ${ }^{[2]}$ Upon calibration using the FTIR technique, sample point $A$ was related to a molar ratio of 1:2 ( $\mathrm{PPX}-\mathrm{CH}_{2}-\mathrm{NH}_{2}: \mathrm{PPX}-\mathrm{COCF}_{3}$ ), sample point $\mathrm{B}$ corresponded to the equimolar ratio of the two building blocks, and sample point $\mathrm{C}$ was related to a $5: 1$ excess of PPX- $-\mathrm{CH}_{2}-$ $\mathrm{NH}_{2}$, at fixed flow rates of $6.5 \mathrm{sccm}$ source $A$ and $32.0 \mathrm{sccm}$ source B. FTIR spectra from other CVD deposition conditions revealed analogous results, indicating different rates of change across the substrate (data not shown). Differences in the chemical side groups of the starting materials had a profound impact on the spreading of the resulting polymer films. This agrees well with a recent study on penetration of CVD coatings into confined microchannels. ${ }^{[27]}$

The FTIR study was complemented by a detailed XPS analysis. In general, XPS provides information about the polymer composition within the top 5-10 $\mathrm{nm}$ of a polymer film. ${ }^{[28]}$ We used XPS in its survey spectrum mode to study the composition of three different CVD films made by gradient co-polymerization on a silicon substrate. Fluorine and nitrogen were selected as reporter atoms for monomers $\mathbf{3}$ and $\mathbf{4}$, respectively. On the basis of these reporter atoms, relative surface ratios were calculated for each film. Figure 3 shows the copolymer concentration profiles based on the XPS results. Upon inspection of the surface composition from Figure 3, gradually changing concentrations can be observed ranging from $26 \%$ of compound 4 at positions A (closest to source 1 ) to $44 \%$ at position B, to $85 \%$ at position $C$ (closest to source 2). A detailed analysis on the basis of 14 data points along the trajectory between the two source inlets revealed a linear change in surface (a)

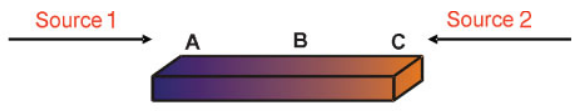

(b)

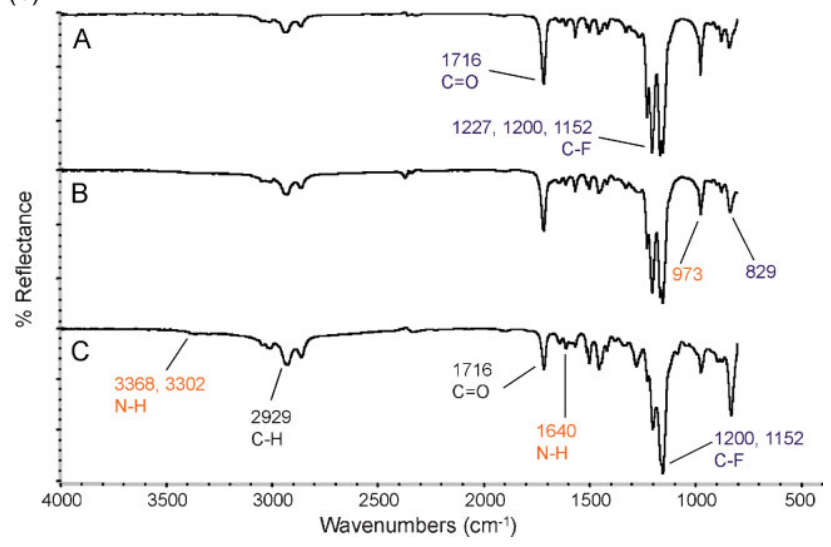

Figure 2. (a) Side view of the CVD sample holder. $A B=B C=7.6 \mathrm{~cm}$. (b) FTIR spectra of CVD copolymers produced from condition 1 outlined in the experimental section. The bulk ratio of CVD copolymer changes with respect to position along the sample holder.

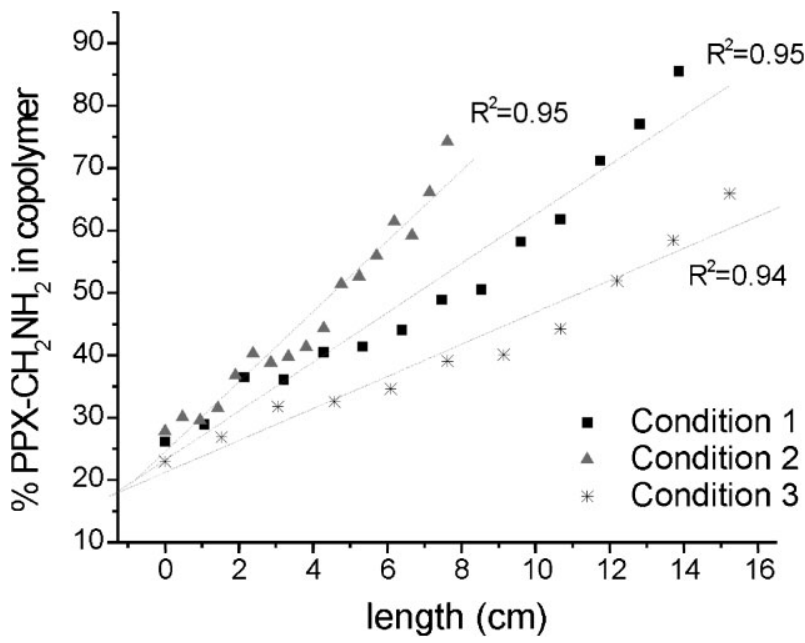

Figure 3. Copolymer compositions along the surface gradient based upon XPS survey spectra. Copolymer ratios are calculated based upon the percentage of fluorine and nitrogen, both characteristic of (1) and (2), respectively. The concentration of aminomethyl groups increases with respect to position along the substrate. The compositional rate of change can be controlled by manipulating argon flow rates and sublimation rates. Process conditions are outlined in the experimental section. 
composition over a distance of $15.2 \mathrm{~cm}$. Simple modulation of the process parameters allowed for controlled manipulation of the gradient compositional slope. To vary the compositional slope of the gradients, cooperative control of argon flow rates and monomer sublimation rates resulted in substantially different gradient slopes as shown in Figure 3. As the ratio of argon flow rates increases $\left(\mathrm{Ar}_{1} / \mathrm{Ar}_{2}\right)$, an increase in compositional slope of the gradients was observed. Fundamentally, effective gradient formation required the deposition zones of both PCP's to sufficiently overlap on the substrate, which was placed onto a cooled sample holder to enhance deposition. If the deposition zones were identical for both PCP's, the sample had to be placed in the center between both sources to obtain optimum deposition. However, it has already been shown for CVD polymerization within microchannels, ${ }^{[27]}$ that trifluoroacetyl-functionalized PCP possesses a higher mobility as compared to the aminefunctionalized dimers, which effectively moves the deposition zone away from the source of the trifluoroacetyl-functionalized PCP and closer to the source of the amino-functionalized PCP. When samples were placed outside of the region of overlapping deposition zones, the resulting gradients were flat and very difficult to control. Moreover, modulation of parameters associated with the CVD polymerization of trifluoroacetyl-functionalized PCP can be expected to be more effective when controlling gradient properties, such as compositional profiles and slopes. Increase in the mass and argon flow of paracyclophane 1, while decrease in the argon flow of paracyclophane $\mathbf{2}$, increased the compositional slope of the gradient, as long as the mass of compound $\mathbf{2}$ was held constant. Because of the different monomer mobility, changes made to flow rates of the amine were small enough, to ensure that the aminomethyl-rich end of the gradient remains unaffected by the increase in trifluoroacetyl-functionalized monomers. Assuming that the sublimation temperatures remain constant, the aforementioned trends in deposition conditions can be used to produce compositional gradients with predictable slopes.

To verify this, three plots are shown in Figure 3, which represent three CVD conditions outlined in the experimental section. Linear trends can be fitted to each plot, each with an $R^{2}$ value of at least 0.94 . In spite of the steeper compositional gradient, the actual range of realizable compositions remains approximately unaltered. The ability to predict surface compositions along the surface gradient solely on the basis of its location with such accuracy is a critical attribute of this gradient synthesis process.

To confirm that free amino and trifluoroacetyl groups are available at the surface in the expected ratios, we reacted the polymer gradients with two fluorescencebased reporter reactants with orthogonal reactivity. Model surface reactions were employed, which followed previously established reactions. ${ }^{[24]}$ First, Atto 655 NHS ester was reacted with the primary amines of $\mathbf{5}$, followed by reaction of the trifluoroacetyl groups of $\mathbf{5}$, with biotin hydrazide to form the corresponding hydrazones. Rhodamine-linked streptavidin was then used to visualize biotin attachment. ${ }^{[2]}$ Figure 4 shows a fluorescence scanning image and normalized fluorescence (a)
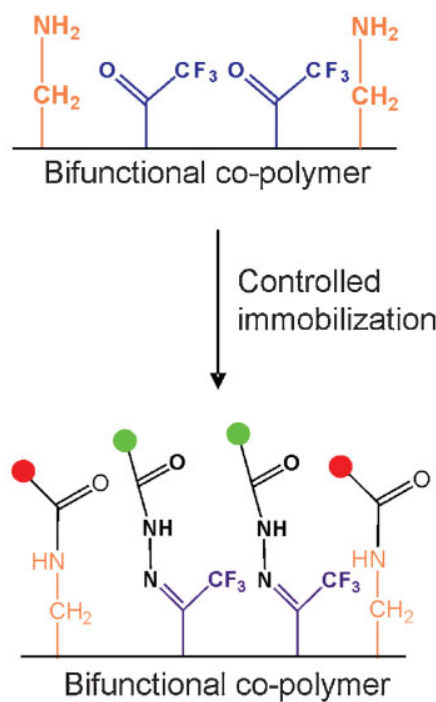

(b)

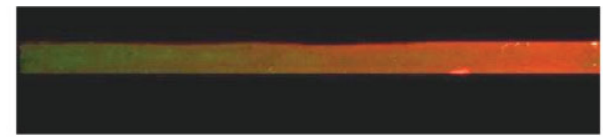

(c)

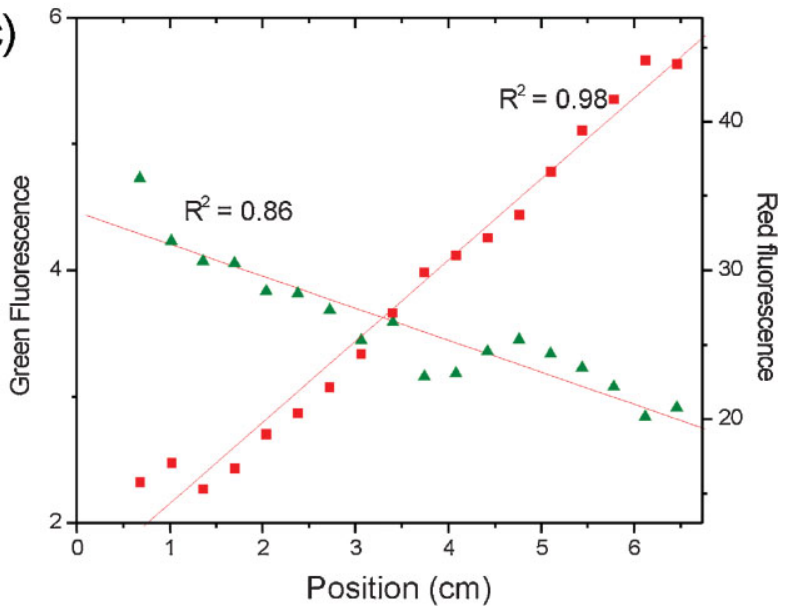

Figure 4. (a) Schematic of the biomolecular immobilization process; (b) fluorescence image and (c) intensity profiles of immobilized fluorescent dyes across a CVD polymer gradient. Both Atto 655 NHS ester (red) and biotin hydrazide with rhodamine-tagged streptavidin (green) reacted over the same regions, demonstrating that the composition gradient can be converted into immobilization gradients. 
profiles for a surface gradient, which was reacted with two fluorescent dyes. To obtain fluorescence profiles, actual fluorescence values within areas of ligand immobilization were divided by the polymer background fluorescence. As the concentration changes across the substrate, the ratio of red versus green fluorescence intensities is altered correspondingly. The fluorescence gradient changed linearly and correlated well with the surface composition ratios obtained by XPS. This is important, because XPS provides information that averages over the outermost 5$10 \mathrm{~nm}$, while the fluorescence titration only reflects chemical groups incidentally available at the surface. Thus, the gradient surfaces not only have well predictable surface composition gradients, but these gradients also correspond to a gradual change in surface reactivity.

\section{Conclusion}

In summary, we have developed a straightforward process for the fabrication of continuous, reactive composition gradients based on vapor-deposited polymer thin films. These poly( $p$-xylylene) coatings consist of a uniform polymeric backbone, with gradually changing functional side groups present at the surface. The films possess a gradient of two reactive functional groups, allowing for independent manipulation of physical and/or chemical properties. By controlling CVD polymerization parameters, such as argon flow rates and sublimation rates; the slope of the compositional gradients can be adjusted, while maintaining a linear trend. Most importantly, we were able to selectively immobilize fluorescently labeled ligands onto the reactive polymer gradients. Not only is CVD polymerization conformal over complex geometries, but the deposition can occur over various substrate materials. In addition, the CVD co-polymerization approach used for preparing the polymer gradients is not limited to the two functionalities discussed herein. A range of different functionalities including alcohols, ${ }^{[30]}$ amines, ${ }^{[31]}$ ketones, ${ }^{[32]}$ esters, ${ }^{[33]}$ alkynes, ${ }^{[34]}$ and aldehydes ${ }^{[35]}$ can be incorporated into these coatings and selecting dimeric precursor(s) with the appropriate functional group may create gradients of a wide range of different functionalities. Future work will include adaptation of the herein described CVD system to accommodate a third source to produce ternary surface gradients. Extension of the available range of gradient concentrations will be another area of further study. CVD composition gradients have the potential to address many common setbacks and limitations of currently employed methods for polymer gradients and may find applications in a range of biotechnological applications including scaffolds for tissue engineering, substrates for microbiological studies or for combinatorial biomaterials screening.
Acknowledgements: JL gratefully acknowledges support from the NSF in form of a CAREER grant (DMR-0449462) and a Major Instrumentation Grant (DMR-0420785). YE acknowledges an NIH/ NIBIB training grant (5 T32 EB005582-02). The authors would like to thank Erdogan Gulari, University of Michigan, for use of the fluorescence scanner, and Hsien-Yeh Chen, University of Michigan, for assistance with the construction of the CVD system.

Received: September 16, 2008; Revised: October 16, 2008; Accepted: October 17, 2008; DOI: 10.1002/marc.200800578

Keywords: biomaterials; coatings; immobilization; surface engineering; surface gradient

[1] H. J. Song, M. Poo, Curr. Opin. Neurobio. 1999, 9, 355.

[2] S. K. W. Dertinger, X. Jiang, Z. Li, V. N. Murthy, G. M. Whitesides, Proc. Natl. Acad. Sci. 2002, 99, 12542.

[3] A. C. von Philipsborn, S. Lang, J. Loschinger, A. Bernard, C. David, D. Lehnert, F. Bonhoeffer, M. Bastmeyer, Development 2006, 133, 2487.

[4] C. A. Parent, P. N. Devreotes, Science 1999, 284, 765.

[5] R. T. Tranquillo, D. A. Lauffenburger, S. H. Zigmond, J. Cell Biol. 1988, 106, 303

[6] H. Mao, P. S. Cremer, M. D. Manson, Proc. Natl. Acad. Sci. USA. 2003, 100, 5449.

[7] R. J. Mrsny, A. T. Gewirtz, D. Siccardi, T. Savidge, B. P. Hurley, J. L. Madara, B. A. McCormick, Proc. Natl. Acad. Sci. 2004, 101, 7421.

[8] L. Ionov, N. Houbenov, A. Sidorenko, M. Stamm, S. Minko, Adv. Func. Mater. 2006, 16, 1153.

[9] [9a] J. C. Meredith, J. L. Sormana, B. G. Keselowsky, A. J. Garcia, A. Tona, A. Karim, E. J. Amis, J. Biomed. Mater. Res. 2003, 66A, 483; [9b] P. Zapata, J. Su, A. J. Garcia, J. C. Meredith, Biomacromol. 2007, 8, 1907.

[10] [10a] J. Genzer, R. R. Bhat, Langmuir 2008, 24, 2294; [10b] T. Winkler, N. Ballav, H. Thomas, M. Zharnikov, A. Terfort, Angew. Chem. Int. Ed. 2008, 47, 7238.

[11] M. K. Chaudhury, G. M. Whitesides, Science 1992, 256, 1539.

[12] T. M. Keenan, C. H. Hsu, A. Folch, Appl. Phys. Lett. 2006, 89, 114103.

[13] N. L. Jeon, S. K. W. Dertinger, D. T. Chiu, I. S. Choi, A. D. Stroock, G. M. Whitesides, Langmuir 2000, 16, 8311.

[14] J. A. Burdick, A. Khademhosseini, R. Langer, Langmuir 2004, 20, 5153.

[15] N. D. Gallant, K. A. Lavery, E. J. Amis, M. L. Becker, Adv. Mater. 2007, 19, 965.

[16] N. Ballav, A. Shaporenko, A. Terfort, M. Zharnikov, Adv. Mater. 2007, 19, 998.

[17] [17a] C. Xu, S. E. Barnes, T. Wu, D. A. Fischer, D. M. DeLongchamp, J. D. Batteas, K. L. Beers, Adv. Mater. 2006, 18, 1427; [17b] I. Luzinov, S. Minko, V. V. Tsukruk, Soft Matter 2008, 4, 714 .

[18] E. Danielson, J. H. Golden, E. W. McFarland, C. M. Reaves, W. H. Weinberg, X. D. Wu, Nature 1997, 389, 944.

[19] M. Zelzer, R. Majani, J. W. Bradley, F. R. A. J. Rose, M. C. Davies, M. R. Alexander, Biomaterials 2007, 29, 172.

[20] [20a] M. S. Kim, G. Khang, H. B. Lee, Prog. Polym. Sci. 2008, 33, 138; [20b] P. Burgos, M. Geoghegan, G. J. Leggett, Nano Letters 2007, 7, 3747; [20c] N. Ballav, S. Schilp, M. Zharnikov, Angew. Chem. Int. Ed. 2008, 47, 1421; [20d] M. Steenackers, A. Kuller, N. Ballav, M. Zharnikov, M. Grunze, R. Jordan, Small 2007, 3, 1764. 
[21] A. Greiner, Trends Poly. Sci. 1997, 5, 12.

[22] V. Murphy, A. F. Volpe, Jr., W. H. Weinberg, Curr. Opin. Chem. Bio. 2003, 7, 427.

[23] [23a] J. D. Whittle, D. Barton, M. R. Alexander, R. D. Short, Chem. Commun. 2003, 14, 1766; [23b] D. E. Robinson, A. Marson, R. D. Short, D. J. Buttle, A. J. Day, K. L. Parry, M. Wiles, P. Highfield, A. Mistry, J. D. Whittle, Adv. Mater. 2008, 20, 1166.

[24] Y. Elkasabi, H. Y. Chen, J. Lahann, Adv. Mater. 2006, 18, 1521.

[25] J. Lahann, D. Klee, H. Höcker, Macromol. Rapid Commun. 1998, 19, 441.

[26] J. Lahann, Polym. Int. 2006, 55, 1361.

[27] H. Y. Chen, Y. Elkasabi, J. Lahann, J. Am. Chem. Soc. 2006, 128 , 374.
[28] "Biomaterials Science: An Introduction to Materials in Medicine", B. D. Ratner, A. S. Hoffman, F. J. Schoen, J. E. Lemons, Eds., Academic, San Diego, CA 1996.

[29] L. Haeusling, B. Michel, H. Ringsdorf, H. Rohrer, Angew. Chem. Int. Ed. 1991, 30, 569.

[30] J. Lahann, R. Langer, Macromolecules 2002, 35, 4380.

[31] J. Lahann, H. Hocker, R. Langer, Angew. Chem. Int. Ed. 2001, 40 , 726.

[32] K. Suh, R. Langer, J. Lahann, Adv. Mater. 2004, 16, 1401.

[33] J. Lahann, I. S. Choi, J. Lee, K. Jensen, R. Langer, Angew. Chem., Int. Ed. 2001, 40, 3166.

[34] H. Nandivada, H. Y. Chen, L. Bondarenko, J. Lahann, Angew. Chem. Int. Ed. 2006, 45, 3360.

[35] H. Nandivada, H. Y. Chen, J. Lahann, Macromol. Rapid Commun. 2005, 26, 1794. 